\title{
Functional Architecture of a Protein-Degradation Machine
}

\author{
Takashi Ishikawa*, Michael R. Maurizi**, Alasdair C. Steven* \\ * Laboratory of Structural Biology, NIAMS; ${ }^{* *}$ Laboratory of Cell Biology, NCI, \\ National Institutes of Health, Bethesda, MD 20892, U.S.A.
}

Protein quality control protects cells from adverse effects of defective or foreign proteins. The intracellular population is monitored for such molecules which are then refolded or eliminated by proteolysis. The latter function is carried out by energy-dependent proteases, enzymes with rings of chaperone-like ATPase subunits stacked on rings of peptidase subunits. The ATPases recognize and unfold substrates, and deliver them to the peptidases for degradation. To elucidate their mode of action, we have studied the ClpAP protease of $E$. coli and its interactions with a model substrate, phage protein RepA. ClpA ATPase consists of two copies of the AAA ATPase domain (D1 and D2), preceded by an N-terminal domain (Fig. 1) and forms a hexamer. ClpP peptidase is a double heptamer: ClpA binds to one or both ends of ClpP. RepA substrates bind initially to sites on the distal surface of ClpA and upon ATPhydrolysis is then translocated $\sim 15 \mathrm{~nm}$ axially into the digestion chamber inside ClpP ([1], Fig. 2a,b,c,e). Our current work is concerned with the disposition of the various protein substrates and enzyme domains during the reaction cycle.

We have calculated a 3 -dimensional model of ClpA at $1.8 \mathrm{~nm}$ resolution $(\mathrm{FSC}=0.5)$ from 1200 tilt pairs of ClpA side-views extracted from the image of ClpAP (Fig. 3). Data were recorded on a CM120 (FEI) with Gatan 626 cryoholder. The reconstruction was done with EMAN [2], using an earlier reconstruction at $2.8 \mathrm{~nm}$ [3] as starting model.

The resulting model has two tiers, corresponding to D1 and D2 respectively (Fig. 4). Each tier is hexagonal with 15 degrees relative axial rotation. The distal face of D2 is in contact with ClpP [4]. Adjacent D2 domains are loosely associated in the ring and have lateral protrusions that may represent the small domains in the distal portion of D2. There is no sign of the Ndomains although they are fully half the size of D1 or D2. Similarly, the N-domains are not evident in averaged side projections (Fig 2d) and only show up as blurred density in a difference image between ClpAP and a mutant complex with deleted N-domains (Fig 2f). From these results, we infer that the $\mathrm{N}$-domain is connected to D1 by a flexible linker. Fig. 4 illustrates two possible modes, in which RepA binds respectively to the $\mathrm{N}$-domains or to a site on the distal face of D1, laterally displacing the N-domains which assemble on the axis in the absence of RepA. Both can account for the broad crescent of terminal density associated with RepA binding ([1]; Fig. 2b, e) and the stoichiometry of one RepA dimer per ClpA hexamer.

\section{References}

[1] T. Ishikawa et al., Proc. Natl. Acad. Sci. 98 (2001) 4328.

[2] S. Ludtke et al., J. Struct. Biol. 128 (1999) 82.

[3] F. Beuron et al., J. Struct. Biol. 123 (1998) 248.

[4] S. K. Singh et al., J. Biol. Chem. 276 (2001) 29429. 

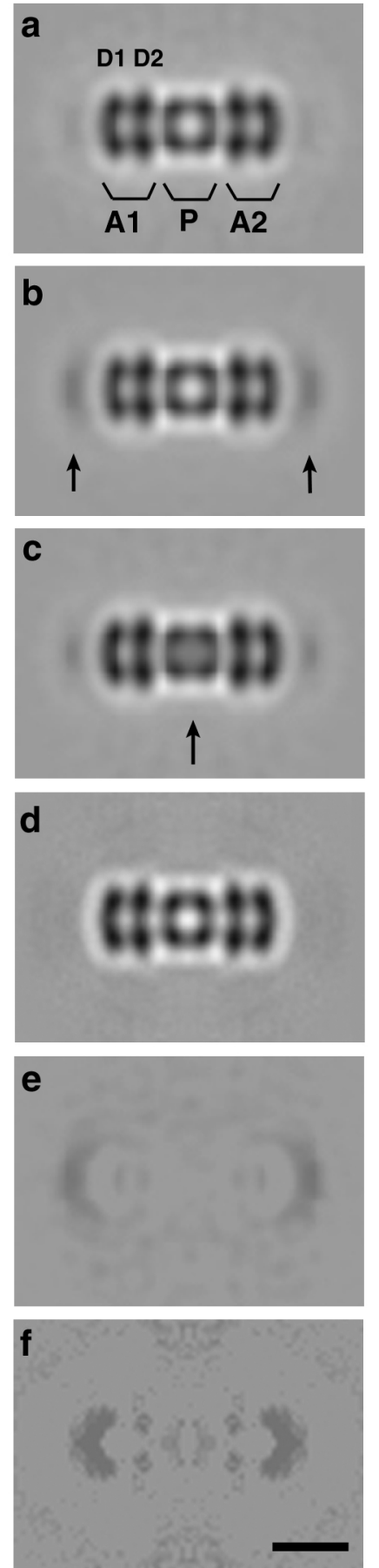

FIG. 2 (a-d) Averaged sideviews: (a) ClpAP+ATP $\gamma$; (b) ClpAP/RepA+ ATP $\gamma$ S; (c) ClpAP/RepA+ATP. The density due to RepA is shown by arrows. (d) ClpAP with ClpA lacking its N-domain. (c, f) Difference maps: (e) between (b) and (a); (f) between (a) and (d). Bar $=10 \mathrm{~nm}$

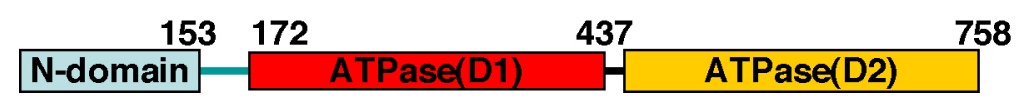

FIG. 1 Schematic diagram of domain structure of ClpA

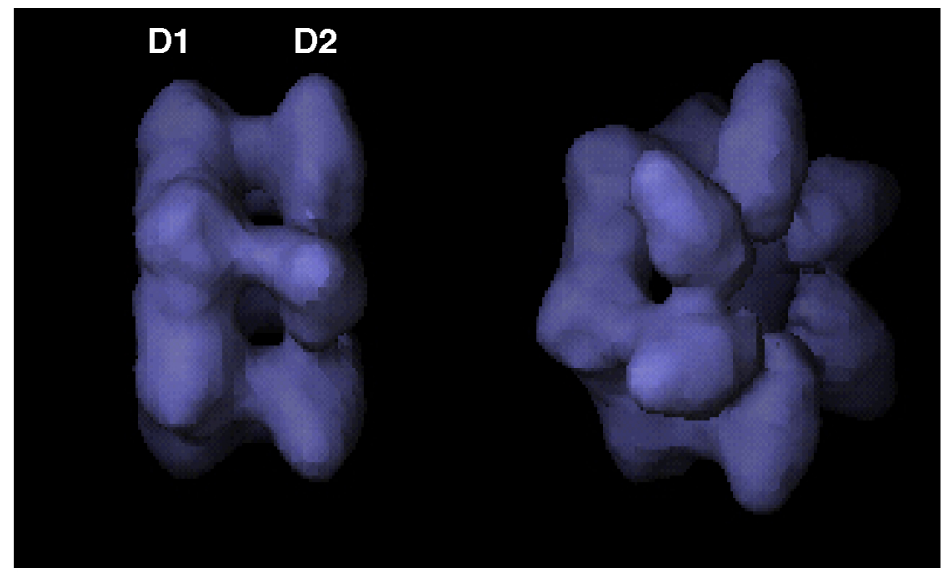

FIG.3 Three-dimensional reconstruction of ClpA hexamer from cryo-electron micrographs. On the left is the same view shown as A1 in FIG.2 On the right is a tilted view.

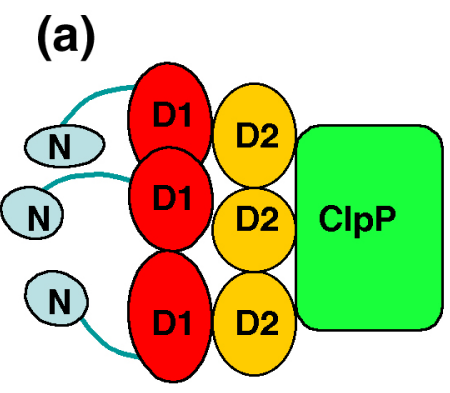

FIG. 4

Schematic representation of the alignment of domains in ClpA (a). Two models of substrate binding are shown $(b, c)$.

(c)
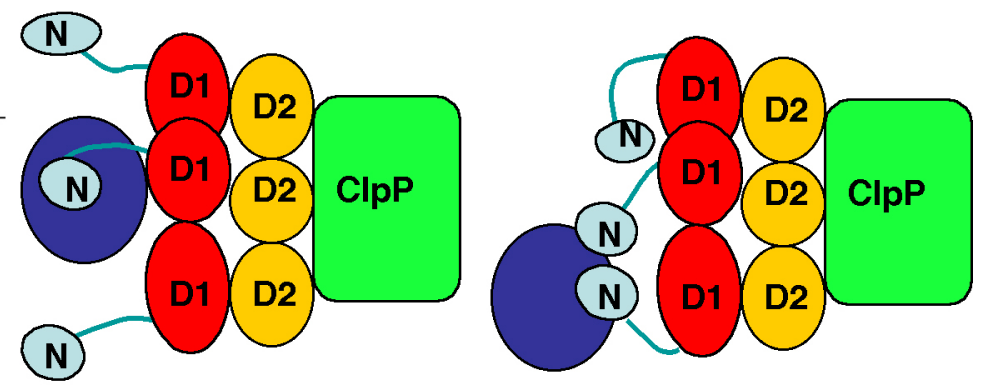